\title{
Théodore Cazaban et Julia Kristeva
}

Un Roumain et une Bulgare en France, deux parcours d'exil

\section{Gina Puică}

\section{(2) OpenEdition}

\section{Journals}

Édition électronique

URL : https://journals.openedition.org/cher/8000

DOI : $10.4000 /$ cher. 8000

ISSN : 2803-5992

Éditeur

Presses universitaires de Strasbourg

\section{Édition imprimée}

Date de publication : 1 décembre 2009

Pagination : 69-86

ISBN : 978-2-35410-006-3

ISSN : 1968-035X

Référence électronique

Gina Puică, « Théodore Cazaban et Julia Kristeva », reCHERches [En ligne], 3 | 2009, mis en ligne le 15 décembre 2021, consulté le 17 décembre 2021. URL : http://journals.openedition.org/cher/8000 ; DOI : https://doi.org/10.4000/cher.8000

\section{(c) $)(1)(2)$}

Ce(tte) œuvre est mise à disposition selon les termes de la Licence Creative Commons Attribution Pas d'Utilisation Commerciale - Partage dans les Mêmes Conditions 4.0 International. 


\title{
Théodore Cazaban et Julia Kristeva Un Roumain et une Bulgare en France, deux parcours d'exil*
}

\author{
GINA PUICĂ \\ Université de Strasbourg
}

es pages qui suivent ne traiteront pas de frontières ou de voisinages
roumano-bulgares. Elles confronteront ce que deux écrivains originaires de Roumanie et de Bulgarie ont pu faire de leur ouverture européenne, en des temps moins propices aux échanges, et comment cette ouverture a pu se répercuter sur les thèmes et les formes de leur œuvre.

En effet, l'occasion m'est donnée ici de revenir sur un sujet qui me préoccupe depuis un certain temps: le positionnement de l'écrivain (ou de n'importe quel créateur, voire d'un individu quelconque) face à son propre exil. Et de réunir ainsi deux facettes opposées, pour ne pas dire extrêmes, de la condition de l'exilé.

Les écrivains concernés par mon article sont Théodore Cazaban et Julia Kristeva. Ils ne se connaissent pas, rien ne semble a priori les rapprocher, sinon le fait d'être tous deux des expatriés d'Europe de l'Est, des écrivains installés en France ayant choisi le français comme langue d'écriture.

Théodore Cazaban (né en 1921) quitte la Roumanie en 1947. Julia Kristeva (née en 1940) part de Bulgarie en 1966. Un jeune homme de 1947, une jeune femme de 1966, quoique provenant d'espaces géopolitiques similaires n'ayant peut-être pas beaucoup évolué entre temps, ne partagent à leur arrivée à Paris ni les mêmes idéaux ni le même projet existentiel. Cazaban est un pur produit de l'idéologie conservatrice-élitiste roumaine de

* Je dédie ce modeste travail à la mémoire de mon frère Codin Puică, disparu alors que ce texte était encore en chantier. 
l'entre-deux-guerres même s'il est plus jeune que les principaux représentants de la célèbre génération d'intellectuels des années trente (Cioran, Eliade). Son existence parisienne est difficilement séparable de la destinée de son pays d'origine où pourtant il ne reviendra jamais. Son œuvre se réduit à un seul livre, tombé dans les oubliettes de l'histoire de la littérature française. Kristeva, quant à elle, est aujourd'hui une personnalité prolifique et protéiforme, mondialement connue, célébrée, exemple achevé d'adaptation à tous les codes et modes de vie, par rapport auxquels elle sait d'ailleurs prendre ses distances, pour mieux exprimer sa personnalité, voire son étrangeté essentielle, fondamentale.

\section{Théodore Cazaban ou l'exil comme détresse}

Théodore Cazaban est un des écrivains de l'exil roumain anti-communiste actuellement redécouvert dans son pays d'origine, à la suite de la chute du régime en 1989. Celle-ci a entraîné un mouvement de récupération des «valeurs de l'exil» largement connues déjà ou plus discrètes. Il fuit la Roumanie en novembre 1947, moins d'un mois avant l'abdication du roi Michel, alors que des changements radicaux se préparent dans son pays et dans le reste de ce qu'on nommera "démocraties populaires", après une invasion soviétique officiellement présentée à l'Est et à l'Ouest comme une libération.

N'acceptant pas d'attendre de voir comment les choses se dérouleront sous les nouveaux auspices, Théodore Cazaban à l'instar de plusieurs compatriotes conçoit comme solution (à ses yeux provisoire) de partir «alerter» l'Occident sur le désastre qui s'est abattu sur sa patrie et les ravages qu'il entraînera dans l'avenir. Habité également de convictions sur une roumanité mythique fortifiée après la création de la «Grande Roumanie» (conséquence du Traité de Versailles entraînant plus que le doublement du territoire), il arrive à Paris à la fin décembre 1947. Il finit par s'y installer (quoique tel ne soit pas son but à l'origine), mais ne semble pas concevoir de projet personnel lié à la France, qu'il aime pourtant en connaisseur, en licencié ès lettres françaises de l'Université de Bucarest. Une fierté familiale aussi le lie à ce pays: ses ancêtres paternels sont de petits commerçants de Carcassonne anoblis par Louis XV. Par-delà l'attrait qu'exerce la capitale française, avec son effervescence culturelle, sur le jeune intellectuel qu'il est, c'est sa mission politique et idéologique initiale qui prévaut, bien qu'elle se change au fil des ans en noble entreprise à finalité illusoire. Là est le hic, comme nous le constaterons. 
Au-delà de petits travaux lui permettant de tout juste survivre dans la capitale française, son existence demeure pendant des années entièrement consacrée à son pays d'origine, tournée vers la Mère-Patrie, happée par le combat contre le régime illégitime instauré à Bucarest. Il est un temps rédacteur du journal dissident publié en France: La Nation Roumaine. Il collabore à Radio Free Europe et à Voice of America (entre 1958 et 1987) ainsi qu'aux nombreux autres (souvent éphémères) organes de presse de l'exil roumain anticommuniste. Il participe au cénacle de Leonid M. Arcade regroupant la plupart des exilés. Il fait paraître des chroniques littéraires (toujours dans les publications de l'exil), s'occupe des activités de l'Institut Universitaire Roumain "Carol I ${ }^{\mathrm{er}}$ " (d'abord comme secrétaire, ensuite comme vice-président).

Cette situation lui fait presque oublier un temps qu'il a débuté (dans la presse) en Roumanie avant de s'exiler, mais elle l'isole d'un milieu d'accueil français inclinant peu à prêter l'oreille au récit d'injustices perpétrées ou se déroulant de l'autre côté du Rideau de fer (donc difficiles à contrecarrer) et aux états d'âme de leurs émissaires.

D'ailleurs, la plupart des exilés roumains se figent à l'époque pour longtemps dans une posture militante de plus en plus schématique, prenant l'engagement «est-éthique» (Monica Lovinescu) pour seule finalité noble, au point de lui sacrifier carrière (littéraire) personnelle, bien-être matériel, visibilité sociale et politique, capital matériel et symbolique. En effet, ce qui caractérise l'exil roumain d'après-guerre, illustré de manière exemplaire par la trajectoire de Cazaban, c'est d'abord son nationalisme, son ethnocentrisme, son inadaptation à la nouvelle donne et au nouvel espace, sa fermeture, son retranchement dans un passé plus ou moins glorieux, dans une «spiritualité » (le mot revient sans arrêt) transcendante mais non moins travaillée idéologiquement, peu prisés par ce $\mathrm{xx}^{\mathrm{e}}$ siècle mouvementé et révolutionnaire, attiré par la modernité dans ce qu'elle suppose de dynamisme, d'immanence, de changement incessant, d'affirmation individuelle. Voilà pourquoi, sauf d'heureuses exceptions confirmant la règle (Mircea Eliade, E. M. Cioran, Eugène Ionesco), la majorité des exilés roumains de cette génération et de la suivante gaspillent leur talent et leur énergie dans de nobles (mais inefficaces) combats politiques et idéologiques, dans un militantisme patriotique les figeant dans la «crispation identitaire» (Tzvetan Todorov), au lieu de leur faire découvrir activement, joyeusement cette autre patrie de l'écrivain que devrait être, selon les mots d'Elias Canetti, tout exil. La mission patriotique, exaspérée dans l'isolement (à la fois 
provoqué et subi) de l'exil, s'effectue en cette période aux dépens de l'œuvre à accomplir.

L'incompréhension du milieu d'accueil face aux positions, actions, doléances, refus de ces exilés de l'Est ne cesse de croître. Au fur et à mesure que les années passent, ces réfugiés politiques deviennent des aigris rabâchant leur rengaine nationaliste sans se rendre à une évidence: les réalités historiques concrètes sont peu propices aux bouleversements géostratégiques européens, mondiaux appelés de leurs vœux. Car par-delà l'inutilité d'un combat unilatéral lassant pour tous, ils n'arrêteront jamais de se fabriquer des arguments en faveur d'une Roumanie immuable, celle de l'entre-deux-guerres (époque présentée comme un âge d'or). Ainsi, malgré l'impression qu'ils laissent et se donnent, aucun effort n'est déployé pour leur permettre de comprendre et modifier (à partir des données du réel) la Roumanie de leur époque. Nulle solution pratique pour relativiser la portée des réalités-mêmes n'est proposée par eux, car ils sont trop peu partisans de la négociation, de la vision en nuances. Cela explique le progressif retournement contre eux de leurs actes et paroles, leur ostracisation intellectuelle, le dialogue de sourds qu'ils installent, entretiennent et enfin, leur incompréhension du sens de l'histoire contemporaine: Mai 1968 ne suscite en eux aucun mouvement de sympathie, son bouillonnement intellectuel ne les touche pas, dans le tournant historique de ces années, ils ne perçoivent que le côté agressif et radical. Tel est donc le contexte dans lequel ces exilés évoluent.

Certes, ils ne délaissent pas la vraie littérature, mais leurs écrits restent en général connus de leurs seuls compatriotes, n'ayant d'impact que sur ceux-ci. Sauf de rares exceptions, ils n'écrivent pratiquement que pour un public roumain exilé ou pour un public virtuel de Roumanie, où ils sont d'ailleurs interdits d'entrée et leurs œuvres proscrites. Leurs milieux sont par conséquent à l'époque un vrai ghetto culturel. Sanda Stolojan, exilée à Paris, note dans son journal, en date du 20 août 1975: «Garder la flamme vivante [c'est-à-dire les souvenirs de Roumanie et le devoir envers ce pays; ma note: G.P.], sans qu'elle vous dévore, c'est notre pari existentiel, à nous qui vivons en état d'exil» (Stolojan, 1999: 9). Or, certains, sinon la plupart d'entre eux, finissent par être bel et bien dévorés par cette flamme...

Dans un contexte rendant difficile l'action concrète aussi bien que la réflexion littéraire, philosophique approfondie - cette dernière surtout exige sa part de détachement -, on peut dire sans risque de se tromper que les 
exilés roumains travaillent moins pour leur pays qu'à leur propre perte. C'est bien le cas de Théodore Cazaban.

Des miracles existent même dans les cas désespérés. Pour Cazaban, le miracle survient avec la parution chez Gallimard, en 1963, de son unique roman Parages, plutôt bien accueilli. Zénith d'une carrière littéraire par ailleurs quasi inexistante bien que son auteur mérite pleinement le titre d'écrivain, c'est bien à un livre d'exil et sur l'exil qu'on a affaire. L'éditeur a d'ailleurs fait inscrire sur son bandeau publicitaire «la grâce de l'exil». À plusieurs égards, c'est aussi le livre d'une vie. C'est en tout cas le lieu parfait de sublimation d'un parcours «exilique», la cristallisation d'une expérience moins collective (communautaire) que personnelle, c'est-à-dire l'exact contraire d'enjeux qui furent ceux de la vie sociale de l'auteur.

Théodore Cazaban se présente volontiers comme un conservateur, voire un réactionnaire dans ses croyances mais il est loin de partager des goûts littéraires vieux jeu. Au contraire, sous cet aspect il se trouve placé plus près qu'il ne le souhaiterait sans doute d'une Nathalie Sarraute, d'un Claude Simon (que d'ailleurs il apprécie). Nathalie Sarraute, à la suite d'autres voix novatrices, mais aussi en avance sur la plupart des représentants du «nouveau roman français", a gentiment dénoncé son époque littéraire comme celle "du soupçon", de l'impossibilité de faire semblant de croire à la vitalité de formes littéraires traditionnelles comme émises par un Dieu omniscient, omniprésent, alors qu'elles sont figées et arbitraires. Théodore Cazaban partage cet avis. Outre ses déclarations, le fait qu'il soit lui-même convaincu de l'inanité (ou de l'épuisement) de la formule du roman axé sur la représentation, imposée par Balzac et les autres auteurs réalistes du $\mathrm{XIX}^{\mathrm{e}}$ siècle, est prouvé par Parages éloigné d'un roman de représentation au déroulement narratif linéaire, par conséquent traditionnel au plan formel... Le livre s'affirme plutôt comme anti-roman, dans la mesure où le refus de la représentation réaliste se lie au refus du récit traditionnel, amenant la «mort» du personnage, de l'intrigue, rendus classiques par une certaine tradition romanesque. Le résultat est un fabuleux roman hybride à intrigue éclatée, mettant en œuvre son propre «faire» (le «discours", la «méthode» sont ici des maîtres mots) et un univers étrange, aux bords de l'irréel. Ainsi, Parages se donne-t-il à lire également comme méta-roman, où le flux de conscience du sujet-narrateur (Cazaban va jusqu'à refuser le nom de personnage-narrateur) veut tout embrasser pour n'en garder à la fin que la quintessence. Les passages descriptifs d'une minutie éliminant presque les objets décrits, alternent avec des considérations sur l'état intérieur d'un sujet 
engagé dans une quête mystérieuse: il fait état de sa condition d'exilé, assez souvent mal dans sa peau.

Ce sujet parlant, narrant, "exilé de tout» (Cazaban, 1963: 79), reconstruisant par bribes le récit de son parcours, vivant dans des chambres d'hôtel, entrant dans les maisons habitées «comme dans un temple», porte en lui les obsessions de son auteur (c'est Cazaban qui l'affirme sans ambages). Il se présente volontiers comme le porte-parole d' "un inconformisme réactionnaire» (Cazaban, 1963: 179), «antipathique»(Cazaban, 1963: 7), souvent teinté d'amertume. De même, il est souvent porté à juger les comportements de ses «semblables déniaisés, critiques, cyniques, sportifs, athés, fornicateurs» (Cazaban, 1963: 123). Dès l'incipit, on le voit excédé, dérangé par le milieu où il se place. À cet exilé, l'autre est intolérable. Aucun émerveillement ne lui paraît possible (excepté celui qu'offre l'amour). Ce n'est d'ailleurs pas la compagnie qu'il cherche mais la solitude, étant même tenté d'abolir (par la pensée) les êtres qui l'entourent. Un auto-examen lui révèle que son état est imputable à l'exil lui faisant prendre conscience de sa distance avec les individus croisés, une situation qui le condamne à un autre exil, intérieur. Une suite d'épreuves l'ont en effet marqué, transformé mais la grâce initiatrice de cet "exil obligé et accepté» l'a fait parvenir à un état d'où il peut considérer sa vie avec maturité, voire maîtriser les choses, faute de les changer comme il souhaiterait.

Le livre a pour thème diffus la déperdition de certaines valeurs et le refus de certaines autres, attitude à rattacher elles aussi aux conceptions de l'auteur, marqué, comme nombre de ses compatriotes et frères d'exil, par la philosophia perennis et les préceptes métaphysiques.

Ainsi, la complexité formelle de Parages répond-elle moins à une volonté de briser les cadres acceptés de la tradition romanesque (alors très à la mode dans l'espace français) qu'à une vision complexe (en profondeur) des réalités, à rebours du sens commun d'alors en littérature, mais aussi de ce qui était en train de se constituer en nouvelle bienséance de la pensée parisienne. Cazaban ne s'est jamais habitué à l'idée que les valeurs (politiques, idéologiques, sociales) qu'il défendait s'étaient effondrées. Il n'a pas voulu assimiler non plus l'idée que le monde était désormais dépourvu de transcendance, que, sur les décombres de la métaphysique ("démantelée» au cours du $\mathrm{xx}^{\mathrm{e}}$ siècle), il fallait construire autre chose. Il n'en constate pas moins les dégâts d'une «déchéance» vue partout autour de lui. C'est pourquoi son roman est avant tout un livre plaçant en son centre (pas forcément visible) une initiation échouée, c'est-à-dire une volonté de 
dépassement avortée. Quant à l'écriture de ce texte, elle aurait représenté pour son auteur un véritable «exercice spirituel» (Cazaban, Badilița, 2002: 220) cependant limité, en intensité comme dans le temps. Cazaban semble croire, en effet, que la littérature ne saurait seule sauver le monde, arrêter celui-ci dans sa course descendante. Est-ce finalement une autre obscure raison qui aurait déterminé Cazaban à se taire après ce premier livre (excepté des livres d'entretiens, des traductions, des recueils de textes de presse publiés en Roumanie après 1990), à se murer dans un exil douloureux autant que fier, lui laissant au moins le loisir de considérer, à l'instar de son narrateur, les «créatures de pure agitation»? (Cazaban, 1963: 195). À moins que ce silence ne soit le signe d'une crise de conscience plus insidieuse: en effet, comment continuer à admettre dans les faits (par l'écriture) que l'on est un moderne, un formaliste alors que le discours assumé publiquement dit le contraire...

\section{Julia Kristeva ou les délices de l'exil}

Dès ses premiers écrits de linguiste-sémiologue, parfois jugés comme relevant d'un technicisme formel exagéré, tels Sémèiôtikè. Recherche pour une sémanalyse (1969), La Révolution du langage poétique (1974), Polylogue (1977), Julia Kristeva se place résolument dans son temps, en plein centre de son temps et du monde qu'elle découvre en même temps qu'elle le conquiert, ambitionnant de les façonner à sa guise. Sa chance est de débarquer à Paris après le dégel, dans un moment de relative ouverture en pleine Guerre Froide, alors que l'Est et l'Ouest se scrutent avec intérêt, voire sympathie, et surtout de ne pas avoir de comptes à régler avec l'Histoire (tel Cazaban), mais à l'inverse de vouloir profiter des bons tours que peut lui jouer cette même Histoire. L'étudiante qu'elle est au départ se transforme au fil des années en redoutable intellectuelle, symbole de cette intelligentsia française admirée et enviée du monde entier.

Sa mobilité et sa réceptivité intellectuelles l'aident beaucoup. Peu de temps après son arrivée en France, elle est parmi les premiers à percevoir et transmettre la nécessité de dépasser le structuralisme alors en vogue, ce dernier se trouvant dans une position incompatible avec son propre refus du sujet. Le rôle de Julia Kristeva dans le basculement des études françaises vers le post-structuralisme (d'abord dans le cadre du séminaire de Roland Barthes) est majeur. Et l'ouverture, la coloration de plus en plus variée de son discours vont aller s'accentuant. Très peu french à ce niveau, elle est une adepte intraitable du décloisonnement, de l'embrassement de plusieurs 
disciplines. Son travail irrigue de nombreux domaines: linguistique, sémiologie, philosophie, psychanalyse, anthropologie et, enfin mais non en dernier lieu, littérature et critique littéraire. Qui plus est, elle écrit beaucoup, faisant ainsi parler son exil, l'offrant à un partage indéfini, son œuvre étant l'expression première de son extraordinaire capacité de transformation. Peu d'écrivains eurent le courage d'embrasser si naturellement de si nombreux domaines de l'esprit, de passer si allègrement de l'un à l'autre, et enfin, de plus en plus à mesure que les années passent - de personnaliser et de singulariser autant leur écriture, en se taillant un chemin personnel à travers la forêt des sciences, au carrefour de plusieurs disciplines. En plus, à partir des années quatre-vingt, les écrits de Kristeva subissent une transformation essentielle, s'allégeant quelque peu du poids de cette préciosité terminologique qui l'a fait reconnaître dans les années soixante, soixante-dix, auprès du groupe Tel Quel, ou de certains de ses maîtres (Roland Barthes), pour emprunter des chemins plus directs, mais non moins ardus.

Mais la vie ne saurait être faite de lectures et de la conception d'ouvrages successifs. L'existence concrète suppose des choix, des engagements. Sous cet aspect également, Kristeva ne manque pas de se placer dès le début à la fine pointe des conduites, dans un mouvement imitatif d'abord, détaché ensuite. Ainsi, dans son premier désir de fusion avec l'espace d'accueil, s'engage-telle dans des combats que peu d'étrangers issus de pays de l'ancien bloc de l'Est auraient intégrés (elle sympathise avec des mouvements gauchistes, rend hommage à Mao). Mais elle ne tardera pas à se dégager des «meutes et de tous les communautarismes", des engagements collectifs, pour privilégier sa singularité, dont elle sait faire un cachet, la marque de sa pensée, de son écriture ainsi que de ses choix de vie, en disciple lointaine de Duns Scot (1266-1308), moine franciscain, logicien, théologien et inventeur du «soi».

Les échecs collectifs lui feront d'ailleurs sans doute prendre conscience $\mathrm{du}$ rôle du sujet et l'amèneront à personnaliser de plus en plus son univers scriptural, à s'étrangéiser donc à l'heure où sa renommée française et internationale grandit, où elle devient une écrivaine et une citoyenne française mais aussi du monde.

Son ambition d'étrangère est de se placer dans un éternel défi face au monde et à ses propres limites, de créer des œuvres en rupture avec les normes, le tout baigné dans l'ironie, voire dans une forme d'apparente légèreté laissant pourtant deviner des abîmes de réflexion. Abordé une première fois en 1990, le genre romanesque, avec les libertés qu'il permet, fait depuis partie de la vie créatrice de Julia Kristeva, toujours sur un mode ironique et subversif. Elle 
rédige ce qu'elle appelle du «thriller métaphysique» (Possessions, Meurtre à Byzance, etc.), ou, plus récemment, des récits inclassables, comme Thérèse mon amour, pour lequel, comme elle l'avouera lors d'une rencontre avec son public de Strasbourg, elle a "dû inventer un genre».

Avant de constater que la singularité est condition de l'éthique (Kristeva, 2007: 24-25), une issue face à la dualité dangereuse du monde, il lui aura fallu se frotter à des choix variés, à ce qui lui était le plus étranger et le plus fascinant à un moment ou à un autre. Au début, s'étrangéiser était pour elle imiter les jeunes intellectuels français progressistes, retourner les impressions politiques acquises dans son pays d'origine pour endosser celles qui la surprenaient, lui faisaient entrevoir des mondes autres, même utopiques, ou bien les mêmes mondes dans une perspective inversée, avant de devenir l'étrangère, la cosmopolite par excellence à force de passer de multiples (autres) frontières. Faut-il souligner le rôle bénéfique de cette expérience et de cette expérimentation?

L'exil de Julia Kristeva est loin d'être simple, bien qu'il soit assez facile de «lire» la façon dont elle l'a vécu et comment celui-ci l'a formée et transformée. Elle se veut une sorte d'apôtre de l'étrangeté. "Nous sommes tous des étrangers » (Kristeva, 2007: 53), voire des «étrangers à nous-mêmes » (titre d'un essai publié en 1988), affirme-t-elle. Et de lancer l'invitation: «Etrangez-vous à vous-mêmes, en vous-mêmes. Apprenez à être Autre» (Kristeva, 2007: passim). Ce thème et certains de ses corollaires ne cessent de venir, de revenir dans ses écrits depuis (au moins) les années quatrevingt, de façon directe ou souterraine. D'origine bulgare, de nationalité française, «adoptée» par les États-Unis, où elle enseigne une bonne partie de l'année, elle semble être aujourd'hui, en effet, l'étrangère par excellence. La transformation continuelle de son parcours (jusqu'à son être) est sa façon à elle de maîtriser son dépaysement.

Rien d'étonnant alors à ce que son premier ouvrage de fiction, Les Samouraïs (1990), porte en son cœur le thème de l'exil. Ce livre complexe, résolument moderne comme écriture et construction d'ensemble (postmoderniste, diraient d'autres) retrace thématiquement la très riche et dynamique atmosphère intellectuelle parisienne des années soixante-dix jusqu'à la fin des années quatre-vingt, notamment la Révolution de Mai 1968 et les parcours des intellectuels-vedettes, ceux des jeunes espoirs rêvant de refaire le monde tout en souhaitant se reconstruire eux-mêmes. Il est avant tout, vu le contexte qui est le nôtre actuellement, une sorte de livre de l'exil (kristévien, tout au moins) sinon un manuel d'exil. 
Largement autobiographique, reconnu comme tel, et plus largement roman à clefs, Les Samouraïs présente le chemin presque initiatique d'Olga, au départ une jeune étudiante d'un pays de l'Est, lequel n'a d'autre nom que «là-bas». Venue à Paris grâce à une bourse d'étude, elle finira par y rester, en se (re)construisant une vie et une brillante carrière, en naissant en quelque sorte une nouvelle fois. Insistons quelque peu sur ce parcours si naturel et néanmoins si spectaculaire.

Débarquée dans le Paris intellectuel en ébullition au milieu des années soixante, Olga est tout de suite fascinée par les jeunes étudiants, professeurs et intellectuels de tout poil qui animent cet espace, chez lesquels elle admire l'enthousiasme intellectuel mêlé d'austérité, "la passion qu'ils mettaient à étudier, à discuter, à creuser les mots et les textes, à confronter des avis subtilement divergents et profondément complices» (Kristeva, 1990: 29). Cette espèce surprenante d'individus est et sera bien la France, semble dire le personnage kristévien, qui ne manque pas de faire sienne leur attitude... Tombée donc sous la fascination française, par ailleurs déjà acquise dans son pays, «là-bas», où la France et la Ville Lumière disent, ou disaient encore, quelque chose, la jeune étudiante est «frappée», voire «déboussolée» "par la proximité immédiate qu'ils lui témoignaient. Plus qu'une acceptation, une sorte de parenté spontanée. Comme s'ils avaient vécu ensemble depuis l'enfance» (Kristeva, 1990: 29). Aucune hostilité ne vient ternir ce rapport entre autochtones et la personne venue d'ailleurs. En tout cas, aucun acte, aucune parole qui instillent en Olga le sentiment de ne pas être à sa place ou quelque tendance auto-défensive.

L'époque étant celle des débats idéologiques les plus spectaculaires, et comme ils ne voulaient pas faire figure de réactionnaires, les nouveaux amis d'Olga voyaient en elle non pas la rescapée d'un espace clos et démuni, rendu exsangue et muet par la terreur stalinienne, mais la messagère de cette «vague de fond " venue de l'Est, qu'ils mythifiaient à souhait, munis de solides, mais utopiques notions issues des avant-gardes esthétiques et des origines de la Révolution. À son tour elle épouse leurs croyances, conceptions, attitudes, avant d'en constater l'échec partiel. Et ce sont ces mêmes raisons largement intellectualisées qui détermineront cette jeunesse à trouver un remède aux malaises et impasses personnels en les faisant se ruer vers la Chine et le chinois. À Paris, la mode était alors, en effet, au mouvement maoïste. Mais par-delà ce phénomène quasi-générationnel, il y avait une question autant intellectuelle que personnelle qui se faisait ainsi jour: «on ne voit loin que de loin, depuis les antipodes» (Kristeva, 1990: 197). 
La Chine devenait le prétexte idéal pour s'arracher à soi-même, à ses habitudes, à ses racines, pour se trouver une "contre-identité», une «étrangeté absolue». Pour Olga, l'épisode chinois est doublement significatif dans la mesure où «longtemps la France avait été sa Chine à elle» (Kristeva, 1990: 196). «Libérée» (le mot est présent dans le texte) de son pays d'origine, pas "réellement intégrée » en France, malgré son adaptation et son envie de réussir, convaincue d'ailleurs qu'elle n'y serait jamais tout à fait adaptée, «même en devenant la mère de dix petits Français», elle trouvait en Chine et en sa culture, après les événements de Mai, une deuxième tentative d'étrangéisation, plus volontaire et plus cérébrale que jamais. Surgissaient en elle désormais des questions à double teneur «exilique» comme: "quels sont les visages extrêmes, les chinoiseries que je sens en moi et que je ne peux formuler?». La Chine devenait ainsi pour elle non seulement une «contreidentité», mais aussi une "anti-origine», car le vœu est clairement formulé: «son expérience d'étrangère devenait l'occasion de se débarrasser de ses origines, au point même de les oublier», sans regret apparent... Car «Olga avait cru comprendre de son éducation ultra-philosophique que les origines sont un atavisme inévitable, mais que le degré de civilisation se mesure à la capacité de les dissoudre» (Kristeva, 1990: 195). Un Théodore Cazaban aurait trouvé sans doute ces mots assassins, lui pour qui l'exil signifiait préservation acharnée des origines...

Mais, forcément, des années plus tard, «l'enthousiasme s'était brisé net chez les camarades» (Kristeva, 1990: 342), Olga et quelques-uns réussirent à "surnager ", c'est-à-dire à préserver une foi, grâce à leur passion du travail (individuel, cela s'entend). L'époque des engagements et des combats collectifs était décidément révolue.

Quoi qu'il en soit, se trouvant dans le «monde libre» (comme on disait alors) après de longues années passées de l'autre côté du Rideau de fer, Olga sait transformer la fatalité historique en leçon de vie. Connaissant le sens (et le non-sens) des frontières géographiques, des limitations arbitrairement idéologiques et culturelles, ayant appris à les déjouer et à en jouer, elle n'a pas trop de mal non plus à partager sur le plan personnel la vie mystérieuse, «tout en frontières» (Kristeva, 1990: 79) et séparations, de l'homme qu'elle aime. La traversée des frontières réelles l'a suffisamment fortifiée pour cela; et la vie lui en fera connaîtra bien d'autres; elle apprendra même à aller à leur rencontre.

Les années passant et les expériences, de même que les difficultés inhérentes à toute vie, se multipliant, l'exil d'Olga s'avère proprement réussi 
dans la mesure où, refusant de se mettre en retrait, de se replier sur ellemême sous le poids du réel, "dans une contemplation solitaire et désabusée du monde», elle continue son «voyage», convaincue que «le monde est fait d'isolements incommensurables", autrement dit, le monde n'est qu'un exil fait de nombreux autres exils, de frontières à l'intérieur d'autres frontières. Pour ce faire, elle se propose et réussit à organiser sa vie « en étoile», cumulant en toutes choses les nouveaux départs, à partir d'un unique "point sensible» (sa force intérieure affermie par l'expérience d'étrangère), sans jamais reculer, utilisant ses angoisses d'étrangère comme moteur de ses actions et l'habitude du risque comme morale existentielle. Son attitude s'avère payante et paraît correspondre à l'" expansion congénitale du monde», à sa tendance «essentielle», à sa "dilatation» (Kristeva, 1990: 285).

Les choses paraissent simples: la réussite de l'exil par le renoncement aux origines et l'ouverture permanente. Or, rien n'est véritablement simple. Et les histoires d'exil ne sont jamais linéaires... Ainsi, la lecture d'un texte un brin circonstanciel que Kristeva écrit en 1994, publié en 1995 dans la revue l'Infini et repris depuis sur son site personnel, nous permet-il quelques remarques supplémentaires. Il s'agit de Bulgarie, ma souffrance, dans lequel l'auteur revient sur son pays d'origine, qui est loin d'être pour elle un sujet de prédilection. Ce texte se présente de prime abord comme un autre hymne aux vertus salvatrices de l'exil. Se félicitant à juste titre de se placer dans une position "dehors-et-dedans" et d'être de la famille des cosmopolites, elle reprend l'idée d'originalité intrinsèque de l'être exilé, déplacé, admettant avec une volupté non-dissimulée qu'«il y a du matricide dans l'abandon d'une langue natale», mais que le désir de "voler plus haut que les parents: plus haut, plus vite, plus fort», c'est-à-dire s'intégrer dans un espace plus prestigieux que ne l'était celui d'origine, peut tout excuser. Elle écrit également noir sur blanc: "Je n'aimerais pas être à votre place [Bulgares de la Bulgarie d'aujourd'hui; ma note: G. P.], et je ne discuterais pas avec ceux qui m'accuseraient d'avoir fui cette difficulté précisément». Les considérations psychanalytico-philosophiques, domaine où elle excelle, alternent avec des retours sur l'histoire, plus lointaine ou plus récente, de son espace d'origine (forcément moins glorieuse que celle de la France), non sans d'incessants rappels de l'histoire française et des remarques quant aux destins des êtres de frontières sillonnant en tout sens le monde du temps présent. Admettant avec condescendance que «nous autres [je souligne: G.P.], orthodoxes, sommes les héritiers d'un nihilisme triomphant», que "nous nous [je souligne: G.P.] sommes mis hors de l'histoire, ce qui est une vertu exorbitante» (c'est ce 
que disait Cazaban, Eliade, et que ne cessent de répéter les conservateurs est-européens de tous bords), elle se lance également dans des remarques plus banales où une exaspération se donne à lire sans trop de ménagements, signe de cet amour-haine ressenti par de nombreux exilés à l'égard de leur pays, qu'ils ne comprennent plus, quelle que soit leur orientation: «Vous [Bulgares] souffrez du chaos, du vandalisme, de la violence. Vous souffrez du manque d'autorité. Vous souffrez de la corruption, de l'absence d'initiative, du laisser-aller que doublent une brutalité sans précédent des individus, l'arrogance de la mafia et les combines des nouveaux riches [je souligne: G.P.]». On aura sans doute remarqué le passage insidieux du nous au vous... Plus finement, elle dénonce la syntaxe lourde, l'excès de néologismes propres aux écrits (traductions, notamment) bulgares parus après la chute du régime communiste. Elle les juge non-avenus, trompeurs faute d'être le reflet d'une «pensée à neuf», d'un "assouplissement de la pensée». Mais n'est-ce pas par le biais des emprunts directs que langues et cultures évoluent depuis que les échanges se multiplient sous l'effet de la globalisation? Toute la culture contemporaine de Sofia, de Paris, de Londres, Bruxelles ou New York n'est-elle pas réalisée à partir des décombres des diverses traditions et de transferts culturels directs parfois brutaux? Plus lointainement et généralement, la loi de l'imitation comme nécessité absolue de l'évolution des sociétés, dont parlait (il est vrai il y a plus d'un siècle!) Gabriel Tarde, reprise dans la Roumanie de l'entre-deux-guerres par le critique et historien Eugen Lovinescu, ne trouve décidément pas en Kristeva une adepte... Et ce d'autant plus qu'elle s'est fait de la défense des apports individuels à la culture une marque propre...

\section{L'originalité de l'exil}

Étudier les comportements "exiliques" amène à la remarque suivante: il n'y a pas que des exils géographiques, il y a aussi des exils historiques. Autrement dit, chez des esprits comme Cazaban, la dimension temporelle de l'expatriation l'emporte: proprement étrangers à leur temps, ils ne tentent pas de l'apprivoiser patiemment, ils ne l'acceptent tout simplement pas. Aussi en subissent-ils la rupture, le choc. Mais pour Kristeva, pleinement ancrée dans son temps, les difficultés sont délicieusement affrontées, conjurées, combattues, abattues. Située dans un dépassement continuel des frontières, elle se place également dans un permanent dépassement de soi et part de son temps, cette fois dans une bienheureuse fuite en avant et non dans cette tentative de récupération d'époques révolues qui est le lot de la plupart des 
exilés. Alors que l'existence de Cazaban ressemble de plus en plus à une mort à soi, celle de Kristeva s'apparente à une renaissance permanente.

Mais par-delà les différences, chez le conservateur Cazaban, comme chez Kristeva, la moderne, la condition «exilique» a apporté sa part d'originalité à la construction de l'individu et de l'œuvre. Chacun de son côté, outre aveuglements et entêtements plus ou moins passagers, a su s'ériger adroitement contre idées reçues et lieux communs. L'intégration est une tout autre affaire.

Pour Cazaban, les idées politiques, sociales n'ont presque pas évolué. Ses idéaux n'ont pas su tenir compte de l'évolution des mœurs, des mentalités, des mille nuances et visages que peuvent prendre Bien et Mal. Chez un adepte tel que lui de la métaphysique dans sa dominante transhistorique, nonévolutive, la situation est compréhensible. Son intransigeance morale tourne ainsi en son contraire. Pour lui, il ne pouvait pas y avoir d'autre Roumanie hors celle de l'entre-deux-guerres, désormais perdue. La poursuite de celleci par les voies détournées de l'exil lui a ainsi fait manquer son intégration sociale en France. Son mépris du communisme l'a déterminé à détester tout mouvement contestataire se revendiquant de gauche. L'aventure représentée par Mai 1968, l'intelligentsia qui s'est affirmée à cette occasion, les courants de pensée novateurs qui en sont issus lui ont échappé en grande partie, quoique sa lucidité et une incontestable capacité de repérage et de contestation des lieux communs ne l'aient jamais vraiment quitté, au même titre que sa bénignité fondamentale. Son amertume s'est accrue du fait de cette incompréhension mutuelle entre un individu minoritaire, tourné vers un passé de plus en plus idéalisé, insaisissable, accaparant et un espace amené à accueillir d'autres doléances et propositions. Vous avez dit intégration?

Replié sur des valeurs de trop peu d'actualité hélas! sinon faussement immuables, Cazaban a emporté la Roumanie avec lui. Kristeva n'a de cesse de se débarrasser du poids de l'héritage bulgare, pour une intégration française fusionnelle à laquelle elle aspirait par-dessus tout, avant de se rendre compte qu'une intégration française absolue se refusera toujours à elle. C'est à partir de là, et grâce à l'engouement massif du moment pour tout ce qui est étranger et éloigné (la Chine de Mao), qu'elle commence seulement à admettre, à désormais privilégier et cultiver son étrangeté essentielle. Après un livre publié sous le nom de son mari (Philippe Joyaux, nom réel de Philippe Sollers) - Julia Joyaux -, elle reviendra définitivement au nom d'auteur qui lui est propre: Julia Kristeva. Tzvetan Todorov, autre Bulgare exilé à Paris naturalisé français, écrit, dans la même optique que 
Kristeva: «J'étais au début un étranger, j'ai aspiré ensuite à être assimilé; une fois ma naturalisation acquise, mon intégration accomplie, je me suis, en effet découvert, 'homme dépaysé'» (Todorov, 2002: 166).

Entre «l'héroïsme masochique des perdants» (Kristeva, 2007: 94) - ces mots de Kristeva s'appliquent aussi à Cazaban - et la désinvolture créatrice et sociale de Kristeva s'ouvre un véritable abîme. Alors que, dans un premier temps au moins, elle se solidarise avec les mouvements gauchistes, Cazaban reproche aux milieux intellectuels français leur complaisance à l'égard des pouvoirs communistes dictatoriaux de l'Europe de l'Est. Face aux malheurs et maladies du pays d'origine, Cazaban a eu raison trop tôt. Dénoncer le régime en 1947 était moins bien vu en Occident qu'à partir des années soixante-dix, après que Soljenitsyne ait mis sa puissance créatrice au service de la dénonciation du Goulag, pour en affirmer les horreurs sur la scène internationale. Kristeva, pour sa part, se souvient de la Bulgarie sur un ton véritablement compassionnel, en 1990 seulement, dans un texte publié dans une revue féminine, Ce que je ne veux pas voir en 1990, repris dans un livre (Kristeva, 2007: 70-73). C'est donc seulement après l'effondrement du mur de Berlin qu'elle parle directement, dans des termes comparables à ceux de Cazaban quarante ans plus tôt, de cette «partie de l'Europe que l'Occident a trop facilement coupée de lui-même et oubliée, depuis quarante-cinq ans déjà», qu'elle se souvient de l'«exaltation dirigée», de la «robotique du collectivisme» qui "camouflaient bien les massacres en coulisses» (Kristeva, 2007: 70-73) dans la Chine de la fin des années soixante où elle pensait trouver alors une salutaire «contre-origine». De crainte de se voir récupérée par les humanistes conservateurs, l'intellectuelle dans le vent n'a vraisemblablement pas osé soutenir les opposants du bloc de l'Est, malgré la solidarité qu'elle devait sentir envers leur sort, aspect indirectement effleuré dans Les Samouraïs (Kristeva, 1990: 143).

Kristeva a décidément voulu dissoudre les démons de l'exil dans l'action, alors que Cazaban n'a pas arrêté de choyer l'«ange de la mélancolie», de préserver sa nostalgie des origines par une attitude contemplative. Kristeva, elle, aura vite remarqué que "la mélancolie n'est décidément pas française» (Kristeva, 2007: 43), ce qui explique son activité fébrile, sa peur des formules figées, sa capacité à renaître, à rebondir, son positionnement constant dans un dépassement de soi. Le cheminement «exilique» cazabanien laisse l'impression de ne pas avancer, de piétiner, celui de Kristeva, se réclamant depuis de nombreuses années d'une modernité analytique dans la lignée de Freud et de Lacan, au cœur de laquelle se place l'expérience du sujet parlant, 
se déploie dans l'élaboration constante de l'œuvre. Le mutisme, l'agraphie de Théodore Cazaban sont un visage lancinant de l'exil, mais ils correspondent également à ce côté métaphysique, réactionnaire, essentialiste qui fut la marque des intellectuels roumains de sa génération, pour qui produire beaucoup équivalait en quelque sorte à se dépenser, à se vider de contenu. Faute d'être l'adepte direct d'une telle philosophie, Cioran s'en faisait aussi l'admirateur (surtout à la fin de sa vie): "Un seul livre aurait suffi. Je n'ai pas eu la sagesse de laisser inexploitées mes virtualités, comme les vrais sages que j'admire, ceux qui, délibérément, n'ont rien fait de leur vie.» (Cioran, 1990: 33).

La conception cazabanienne de l'exil se place dans le droit fil de l'héritage grec. En effet, bien avant que l'exil ne soit accepté, voire recherché, comme le démontrent l'errance, le vagabondage du héros picaresque, cher à Kristeva, qui puise dans ce modèle son apparente légèreté, son ironie), pour les Grecs, l'«exil était le châtiment suprême» (Liiceanu, 1983: 107). Ces derniers recherchaient par-dessus tout la quiétude du foyer, compris comme aspiration à l'ordre immuable, sécurisant, l'égalité avec soi-même. Mais cette conception nostologique s'avère résistante bien au-delà de l'Antiquité grecque. Comme le remarquait G. Liiceanu, chaque période de transition, donc de désordre, est marquée par l'obsession du retour à un giron (ordre, maison, patrie) idéal (óikos). Mais jamais une époque n'aura été aussi incertaine de la stabilité du monde que la nôtre. Cazaban et Kristeva l'ont compris chacun à sa façon, d'où l'impression de vivre en tout lieu, toute circonstance en état d'exil, un exil permanent, généralisé. Ceci amène le même auteur à écrire dans l'article cité que nous assisterions à une «impossibilité généralisée du nostos», que nous sommes donc condamnés à vivre dans l'«a-nostalgie».

Il est fascinant et instructif de constater à quel point le poids de l'histoire et le contexte d'origine influent sur le cours d'une vie, créant des frontières psychologiques et sociales abyssales. Entre Cazaban, le «chevalier résigné » ${ }^{1}$, et Kristeva, le «samouraï» en révolte et combat permanents, fascinée par le lointain et le changement, il y a de multiples façons d'être étranger. Si Cazaban peut être considéré du point de vue des attitudes sociales comme un prototype d'exilé roumain, Kristeva est un cas à part, exceptionnel dans l'ensemble des écrivains bulgares expatriés. Ni l'un ni l'autre ne sauraient

1 Nicolae Florescu et Ileana Corbea ont co-signé un livre d'entretiens avec un certains nombre d'écrivains roumains de l'exil, dont Cazaban, intitulé Resemnarea cavalerilor [ $\mathrm{La}$ résignation des chevaliers] cité en bibliographie. 
en tout cas être des symboles de l'échec ou de la réussite de l'exil. Ni l'un ni l'autre n'aimerait sans doute se savoir lu(e) respectivement dans le miroir de l'autre à l'aune de valeurs qui ne sont pas les siennes. Mais l'exercice en a valu la peine, nous ayant permis de voir comment des couches successives de l'histoire façonnent les individus, leur choix de vie et comment la rencontre de l'autre peut être enrichissement ou, au contraire, affrontement plus ou moins violent.

Quelque peu toujours en marge, l'exilé fait rarement le choix d'un parcours sans heurt. Son parcours est une continuelle épreuve, l'épreuve même de l'étranger. À bien y regarder, les cas de Cazaban et de Kristeva illustrent respectivement les deux facettes opposées sinon extrêmes de l'exil: la fidélité de principe (sinon réelle) à la patrie d'origine, l'oubli de celle-ci pour une renaissance dans la nouvelle patrie et la nouvelle langue, dans de nouvelles patries et de nouvelles langues.

\section{Bibliographie}

Cazaban, Théodore, 1963, Parages, Paris, Gallimard.

Cazaban, Théodore (Theodor) en dialogue avec Bădiliță, Cristian, 2002, Captiv în lumea liberă, Cluj, Ed. Echinox.

Cazaban, Theodor, 2002, Eseuri şi cronici literare, Bucarest, Ed. Jurnalul literar, coll. «Critica».

Cioran, 1990, Entretiens avec Sylvie Jaudeau, Paris, José Corti.

Corbea, Ileana, Florescu, Nicolae, 2002, «Cu Theodor Cazaban invocînd "îngerul melancoliei”...», in Resemnarea cavalerilor, p. 33-46, Bucarest, Ed. Jurnalul literar.

Florescu, Nicolae, 1998, Intoarcerea proscrişilor. Reevaluări critice ale literaturii exilului, Bucarest, Ed. Jurnalul literar.

Kristeva, Julia, 1990, Les Samouraïs, Paris, Gallimard, Folio, nº 2351.

Kristeva, Julia, 1991, Etrangers à nous-mêmes, Paris, Gallimard, Folio essais, nº 156.

Kristeva, Julia, 2007, Seule une femme, Editions de l'Aube.

Kristeva, Julia, 1995, Bulgarie, ma souffrance, http://www.kristeva.fr/bulgarie.html

Lapierre, Nicole, 2004, Pensons ailleurs, Paris, Gallimard, Folio Essais, n 482.

Liiceanu, Gabriel, 1983, Repères pour une herméneutique de l'habitation, in Constantin

Tacou, Les symboles du lieu, l'habitation de l'homme, Paris, Editions de l'Herne, p. $105-116$.

Todorov, Tzvetan, 2002, Devoirs et délices. Une vie de passeur. Entretiens avec Catherine Portevin, Paris, Editions du Seuil, coll. «Points».

Sarraute, Nathalie, 1956, L'ère du soupçon, Essais sur le roman, Paris, Gallimard. 
Stolojan, Sanda, 1999, Au balcon de l'exil roumain à Paris, Paris, Editions L'Harmattan. 